\title{
Output Feedback Control for a Class of Nonlinear Systems with Actuator Degradation and Sensor Noise
}

\author{
Weiqing Ai, Zhenli Lu, ${ }^{\dagger} \quad \mathrm{Bin} \mathrm{Li}^{\ddagger} \quad$ Shumin $\mathrm{Fei}^{\S}$
}

\begin{abstract}
This paper investigates the output feedback control problem of a class of nonlinear systems with sensor noise and actuator degradation. System nonlinear terms satisfy the Lipschitz condition while the precise information of sensor noise and effectiveness of actuator are unknown. Firstly, by using the descriptor observer approach, the origin system is transformed into a descriptor system. On the basis of the descriptor system, a novel Proportional Derivative (PD) observer is developed to asymptotically estimate sensor noise and system state simultaneously against actuator degradation. Then, by modeling actuator degradation and designing an adaptive law to estimate the effectiveness of actuator, an adaptive observerbased controller is constructed to ensure that system state can be regulated to the origin asymptotically in the presence of sensor noise and actuator degradation. Finally, the design scheme is applied to address a flexible joint robot link problem.
\end{abstract}

Key words: Nonlinear system; output feedback; observer design; sensor noise; actuator degradation

\section{Introduction}

Various malfunction or imperfect behavior always occur in the realistic control system. This kind of phenomenons can be derived from the unexpected variations in external surroundings and sudden changes in signals, which are categorized as sensor noise or actuator fault. When sensor noise occurs in measurement channel or actuator fault occurs in input channel, they will cause serious degradation of the system performance, and may even render the control system unstable. Hence, the research in this filed is challenging and attracts many interest.

\footnotetext{
*Weiqing Ai(Correspondence author), School of Electrical Engineering and Automation, Changshu Institute of Technology, 215500, Changshu, China. State key Laboratory of Robotics, Shenyang Institute of Automation, Chinese Academy of Sciences,Shenyang,110014,China. E-mail: awq-archer@hotmail . com

${ }^{\dagger}$ Zhenli Lu, School of Electrical Engineering and Automation, Changshu Institute of Technology, 215500, Changshu, China. State key Laboratory of Robotics, Shenyang Institute of Automation, Chinese Academy of Sciences,Shenyang,110014,China. Email: zhenlilu@cslg.cn

${ }^{\dagger}$ Bin Li, State key Laboratory of Robotics, Shenyang Institute of Automation, Chinese Academy of Sciences,Shenyang,110014,China. E-mail: libin@sia.cn

${ }^{\S}$ Shumin Fei, School of Automation, Southeast University, Nanjing, Jiangsu 210096, China. E-mail: smf ei@seu .edu .cn
} 


\title{
Output Feedback Control for a Class of Nonlinear Systems with Actuator Degradation and Sensor Noise
}

\begin{abstract}
This paper investigates the output feedback control problem of a class of nonlinear systems with sensor noise and actuator degradation. System nonlinear terms satisfy the Lipschitz condition while the precise information of sensor noise and effectiveness of actuator are unknown. Firstly, by using the descriptor observer approach, the origin system is transformed into a descriptor system. On the basis of the descriptor system, a novel Proportional Derivative (PD) observer is developed to asymptotically estimate sensor noise and system state simultaneously against actuator degradation. Then, by modeling actuator degradation and designing an adaptive law to estimate the effectiveness of actuator, an adaptive observerbased controller is constructed to ensure that system state can be regulated to the origin asymptotically in the presence of sensor noise and actuator degradation. Finally, the design scheme is applied to address a flexible joint robot link problem.
\end{abstract}

Key words: Nonlinear system; output feedback; observer design; sensor noise; actuator degradation

\section{Introduction}

Various malfunction or imperfect behavior always occur in the realistic control system. This kind of phenomenons can be derived from the unexpected variations in external surroundings and sudden changes in signals, which are categorized as sensor noise or actuator fault. When sensor noise occurs in measurement channel or actuator fault occurs in input channel, they will cause serious degradation of the system performance, and may even render the control system unstable. Hence, the research in this filed is challenging and attracts many interest.

Since sensor noise is non-vanishing with respect to system state and usually unknown, the design of observer and controller is unable to use the exact value of system output. To reduce or even eliminate the effect of sensor noise on the system performance, many design schemes were developed. In [1], the authors proposed a measurement feedback controller for a class of feedforward nonlinear systems. The controller was coupled with a low-pass filter to attenuate the effect of sensor noise and rendered the system state ultimately bounded. Compared with [1], the condition of sensor noise in [2] was not limited to deterministic 
periodic signals and allowed to be a random signal having nonzero-mean. By introducing a notion of virtual state and using high-gain observer technique, the authors proposed a scaled output feedback controller for a class of feedforward system to render that all the system states and output remained to be bounded in the presence of sensor noise. Meanwhile, based on the high-gain observer technique, discrete high-gain observer[3], linear switched high-gain observer[4], nonlinear high-gain observer[5] and adaptive high-gain observer[6] were designed respectively for a class of nonlinear systems in the presence of sensor noise to ensure that the estimated error was ultimately bounded. In [7, 8], the author further considered a more general nonlinear systems whose nonlinear terms are not limited to the Lipschitz condition. A traditional full-order nonlinear observer is developed to guarantee a desired performance.

There exist some drawbacks in the design schemes of $[1,2,3,4,5,6,7,8]$, that is, the effect of sensor noise on the system performance is only reduced not eliminated and sensor noise can not be reconstructed. Besides, actuator fault is not considered. To overcome the aforementioned main drawbacks, by introducing an augmented vector to construct an augmented system, the work of [9] proposed a novel observer design scheme coupled with two observers: (i) a high-gain sliding mode observer aims to get the exact estimates of both the auxiliary outputs and their derivatives in a finite time; (ii) an adaptive robust sliding mode observer aims to asymptotically estimate system state and reconstruct actuator fault and sensor noise for a class of nonlinear systems simultaneously. The work of [10] modified the observer design scheme in [9] to avoid using the information of time derivatives of the auxiliary outputs. While in $[11,12]$, by decoupling the origin systems and designing two sliding mode full-order observers for two decoupled subsystems, respectively, system state, sensor noise and actuator fault can be estimated simultaneously. Although the effect of sensor noise and actuator fault on the system performance is eliminated, the complexity of calculation and the dimension of system are increased for the sake of two observers proposed in $[9,10,11,12]$. To further simplify the design procedure, the work of $[15,16,17,18,19,20]$ developed a novel descriptor sliding mode approach to exactly estimate system state, actuator fault and sensor noise simultaneously, which is inspired by the descriptor observer approach in $[13,14]$. Based on the estimated state, an observer-based fault-tolerant controller was constructed to stabilize the resulting fault system. Recently, by employing backstepping technique, [21] proposes a novel adaptive backstepping-based fault-tolerant control method which can tolerate three additive (bias,drift and loss of accuracy) and one multiplicative (degradation) sensor faults for a class of MIMO uncertain nonlinear systems. While in [22], a particular fault detection sliding mode observer is designed for the augmented system formed by the nonlinear system with 'observer unmatched' uncertainties and incipient sensor faults. By using Linear Matrix Inequalities (LMI) and line filter techniques, the generated residuals are guaranteed to be robust to uncertainties and that sliding motion is not 
destroyed by faults.

From the point of system model of fault control system, actuator fault can be categorized into 'additive actuator fault' and 'multiplicative actuator fault'. Additive actuator fault is added into the control input channel, which can be viewed as a matched disturbance. The actuator fault considered in the aforementioned papers is all limited to additive actuator fault. Multiplicative actuator fault leads to sudden changes in control parameters or gains of system model. Actuator degradation is involved in the multiplicative actuator fault. It is well-known that actuator degradation in actual physical systems is usually inevitable and often yields performance degradation or even instability. It is more difficult to be dealt with because the actuator degradation is dependent of control input, instead of viewed as an independent disturbance. In the past decades, various reliable control methodologies have been proposed to address the control problem of different systems with actuator fault, see $[23,24,25,26,27,28]$ and the references therein. Recently, [29] addresses a fault detection problem of switched systems with servo inputs and sensor stuck faults. By designing a switching law and its associated fault detection filters, the arbitrarily small sensor stuck faults, including outage faults can be detected in finite-frequency domain. The levels of sensitivity and robustness are measured in terms of the finite-frequency $H_{\infty}$ index and $L_{2}$-gain. In [30], sector condition method and weighting fault signal approach are employed to deal with a fault detection problem for a class of uncertain nonlinear Markovian jump stochastic systems with mode-dependent time delays and sensor saturation. [31] addresses a new model-based fault detection, estimation, and prediction scheme for linear distributed parameter systems with both multiplicative actuator and sensor faults. The design scheme can be concluded as: an observer is proposed by using the PDE representation and the detection residual is generated by taking the difference between the observer and the physical system outputs; a fault is detected by comparing the residual to a predefined threshold.

Compared with the aforementioned results, this paper aims to address the problem of nonlinear systems with actuator degradation and sensor noise. Firstly, the model of actuator degradation is presented. Then, by using the descriptor observer approach, the original system is transformed into a descriptor system, on the basis of which a novel PD observer is developed with an adaptive law estimating the effectiveness of actuators. Finally, by using LMI technique, an adaptive observer-based controller with appropriate choices of design parameters is constructed to render that system state and sensor noise are asymptotically estimated simultaneously and system state is regulated to the origin asymptotically. The main contributions of this paper can be concluded as follows: (i) A novel PD observer approach is presented to reconstruct system state and sensor noise simultaneously with an adaptive law estimating the effectiveness of actuators; (ii) Compared to the aforementioned observers based on the sliding mode technique, the design procedure is 
simple and avoids discussing the reachability of sliding mode surface; (iii) The proposed adaptive observerbased controller is effective for any nonlinear systems satisfying the Lipschitz condition, instead of limited to triangular structure systems.

Notations: Throughout the paper, $\|\cdot\|$ and $|\cdot|$ denote, respectively, the Euclidean norm and 1 -norm of a vector. Given a symmetric matrix $X, X>0$ denotes that $X$ is a positive definite matrix. $X^{T}$ denotes its transpose, $X^{-1}$ denotes its inverse, $\operatorname{Trace}\{X\}$ denotes its trace, $\lambda(X)$ denotes its eigenvalue, $\operatorname{rank}[X]$ denotes its rank. $\operatorname{Re}[\cdot]$ denotes the real part of a complex number. $\operatorname{sign}(\cdot)$ denotes its sign. $\operatorname{Proj}[a, b]\{c\}$ denotes the projection operator whose role is projecting $c$ to the interval $[a, b]$.

\section{Problem formulation}

Consider a class of nonlinear systems in the presence of sensor noise described as follows

$$
\begin{aligned}
& \dot{x}(t)=A x(t)+B u(t)+g(x(t)), \\
& y(t)=C x(t)+f_{s}(t),
\end{aligned}
$$

where $x(t) \in R^{n}$ is the state vector, $u(t) \in R^{m}$ is the control input, $y(t) \in R^{p}$ is the measurement output. $A \in R^{n \times n}, B \in R^{n \times m}$ and $C \in R^{p \times n}$ are known constant real matrices. $g(x(t)) \in R^{n}$ is a continuous nonlinear vector function satisfying the Lipschitz condition. $f_{s}(t) \in R^{p}$ is the unknown sensor noise vector.

In realistic control system, the actuator degradation is usually inevitable. Inspired by [27, 32], the actuator degradation is described as

$$
u^{F}(t)=\rho u(t), \rho=\operatorname{diag}\left(\rho_{1}, \cdots, \rho_{m}\right),
$$

where $\rho_{k}, k=1, \cdots, m$ is an unknown constant satisfying $0 \leq \underline{\rho}_{k} \leq \rho_{k} \leq \bar{\rho}_{k} \leq 1 . \underline{\rho}_{k}$ and $\bar{\rho}_{k}$ are the known lower and upper bounds of $\rho_{k}$, respectively. Thus the system dynamic of (1) with actuator degradation and sensor noise is modeled as

$$
\begin{aligned}
\dot{x}(t) & =A x(t)+B \rho u(t)+g(x(t)), \\
y(t) & =C x(t)+f_{s}(t) .
\end{aligned}
$$

The objective of this paper is to propose a novel observer design scheme against sensor noise and actuator degradation such that sensor noise and system state can be globally estimated simultaneously. Moreover, an adaptive observer-based controller is constructed to ensure that system state can be regulated to the origin asymptotically. 
In what follows, some important assumptions and a lemma are provided which play key roles for the design scheme. The time variable $t$ will be omitted for the sake of simplicity.

Assumption 2.1 [33] The matrices $C$ and $B$ satisfy the following relation

$$
\operatorname{rank}[C B]=\operatorname{rank}[B]=m
$$

Assumption 2.2 [33] For every complex number s with non-negative real part, the following rank condition holds

$$
\operatorname{rank}\left[\begin{array}{cc}
s I_{n}-A & B \\
C & 0
\end{array}\right]=n+m .
$$

Assumption 2.3 [17] The sensor fault vector $f_{s}$ satisfies the following norm bounded constraint

$$
\left\|f_{s}\right\| \leq \alpha
$$

where $\alpha>0$ is a known constant.

Assumption 2.4 [12] The nonlinear function $g(x)$ satisfies the Lipchitz constraint, that is, $\forall x, \hat{x} \in R^{n}$,

$$
\|g(x)-g(\hat{x})\| \leq L_{f}\|x-\hat{x}\|
$$

where $L_{f}>0$ is the Lipchitz constant.

Lemma 2.1 [17, 34] Given a pair of matrices $(A, C)$ with $A \in R^{n \times n}$ and $C \in R^{p \times n}$, the following two conditions are equivalent. (i) The matrix $A$ is stable. (ii) If the pair $(A, C)$ is detectable, then the Lyapunov equation $A^{T} P+P A=-C^{T} C$ has a unique solution.

Remark 2.1 It is clear that Assumption 2.1 and Assumption 2.2 are known as the minimum phase condition. As pointed out in [33], these two assumptions are necessary and sufficient conditions for the existence of (26), (27), (28) and (29). Assumption 2.3 means that the considered sensor noise signal is bounded such as sinusoidal signals. The bound of sensor noise will be used for the design of the novel observer. Assumption 2.4 ensures the existence and uniqueness of system solution. It is a natural condition which can be found in mechanical systems or circuit systems. 


\section{System transformation and observer design}

To begin the presentation of the novel observer, some augmented variables and matrices are first introduced as follows

$$
\begin{aligned}
& \bar{x}=\left[\begin{array}{l}
x \\
f_{s}
\end{array}\right], \bar{A}=\left[\begin{array}{cc}
A & 0 \\
0 & -I_{p}
\end{array}\right], \bar{B}=\left[\begin{array}{c}
B \\
0_{p \times m}
\end{array}\right], \bar{C}=\left[\begin{array}{ll}
C & I_{p}
\end{array}\right], \\
& E=\left[\begin{array}{cc}
I_{n} & \\
& 0_{p \times p}
\end{array}\right], \bar{N}=\left[\begin{array}{c}
0_{n \times p} \\
I_{p}
\end{array}\right], F=\left[\begin{array}{c}
I_{n} \\
0_{p \times n}
\end{array}\right] .
\end{aligned}
$$

Therefore, the original system (3) can be rewritten as

$$
\begin{aligned}
E \dot{\bar{x}} & =\bar{A} \bar{x}+\bar{B} \rho u+F g(x)+\bar{N} f_{s}, \\
y & =\bar{C} \bar{x} .
\end{aligned}
$$

The above system (9) is called as a descriptor system in [17], whose states are constituted of system state $x$ and sensor noise $f_{s}$. By defining

$$
\bar{L}_{D}=\left[\begin{array}{ll}
\bar{L}_{D_{1}}^{T} & \bar{L}_{D_{2}}^{T}
\end{array}\right]^{T}=\left[\begin{array}{ll}
0_{p \times n} & W^{T}
\end{array}\right]^{T}
$$

where $W=\operatorname{diag}\left\{g_{1}, \cdots, g_{p}\right\}$ and $g_{i}>0$ for $i=1,2, \cdots, p$, it can be verified that $\bar{S}=E+\bar{L}_{D} \bar{C}$ is non-singular and $\bar{C} \bar{S}^{-1} \bar{L}_{D}=I_{p}, \bar{A} \bar{S}^{-1} \bar{L}_{D}=-\bar{N}$ hold.

Thus, on the basis of the above properties, a novel PD observer for descriptor system (9) is designed as

$$
\left\{\begin{array}{l}
\bar{S} \dot{\bar{z}}=\left(\bar{A}-\bar{L}_{p} \bar{C}\right) \hat{\bar{x}}+\bar{B} \hat{\rho}(t) u+\bar{L}_{p} y+F g(\hat{x})+\bar{N} u_{s} \\
\hat{\bar{x}}=\bar{z}+\bar{S}^{-1} \bar{L}_{D} y
\end{array}\right.
$$

where $\bar{z} \in R^{n+p}$ is the state vector of the dynamic system (11), $\hat{\bar{x}}=\left[\hat{x}^{T}, \hat{f}_{s}^{T}\right]$ is the estimation of $\bar{x}, \bar{L}_{p}$ is an appropriate matrix to be determined later, $u_{s}$ is a discontinuous input to be designed against the effect of sensor noise, $\hat{\rho}=\operatorname{diag}\left(\hat{\rho}_{1}, \cdots, \hat{\rho}_{m}\right)$ is determined by an adaptive law, which is designed to dominate the effect of actuator degradation.

Remark 3.1 In this remark, the non-singular property of $\bar{S}=E+\bar{L}_{D} \bar{C}$ and the equations $\bar{C} \bar{S}^{-1} \bar{L}_{D}=I_{p}$, $\bar{A} \bar{S}^{-1} \bar{L}_{D}=-\bar{N}$ are proved. Since the descriptor system matrices $E$ and $\bar{C}$ have the following property:

$$
\operatorname{rank}\left[\begin{array}{c}
E \\
\bar{C}
\end{array}\right]=\operatorname{rank}\left[\begin{array}{cc}
I_{n} & 0 \\
0 & 0_{p \times p} \\
C & I_{p}
\end{array}\right]=n+p,
$$


with $\bar{L}_{D}=\left[\begin{array}{ll}0_{p \times n} & W^{T}\end{array}\right]^{T}$ in mind, it can be calculated that

$$
\operatorname{rank}[\bar{S}]=\operatorname{rank}\left[E+\bar{L}_{D} \bar{C}\right]=\operatorname{rank}\left[\begin{array}{cc}
I_{n} & 0 \\
W C & W
\end{array}\right]=n+p
$$

Thus, it is obvious to know that $\bar{S}$ is non-singular. Moreover, by a direct calculation, one has

$$
\bar{S}^{-1}=\left[\begin{array}{cc}
I_{n} & 0 \\
-C & W^{-1}
\end{array}\right]
$$

which leads to

$$
\bar{C} \bar{S}^{-1} \bar{L}_{D}=I_{p}, \quad \bar{A} \bar{S}^{-1} \bar{L}_{D}=-\bar{N} .
$$

Remark 3.2 By a direct calculation, the novel observer (11) can be transformed into an equivalent observer (23). It is obvious that (23) is a PD observer with $\bar{L}_{p}$ being a proportional gain matrix and $\bar{L}_{D}$ being a derivative gain matrix. As pointed out in [13], the present observer (11) is actually a modified form of the PD observer (23). The derivative term of the output $y(t)$ does not appear in the modified PD observer (11).

Thus, it is more applicable in practical systems than the PD observer (23).

\section{Determination of gain matrix $\bar{L}_{p}$}

In this section, the design procedure of $\bar{L}_{p}$ is provided and under the appropriate choice of $\bar{L}_{p}$, the matrix $\bar{S}^{-1}\left(\bar{A}-\bar{L}_{p} \bar{C}\right)$ is proved to be a Hurwitz matrix.

From the definitions of $\bar{S}$ and $\bar{A}$, for every complex number $s$ with non-negative real part, one has

$$
\operatorname{rank}\left[\begin{array}{c}
s I_{n+p}-\bar{S}^{-1} \bar{A} \\
\bar{C}
\end{array}\right]=\operatorname{rank}\left[\begin{array}{cc}
\bar{S}^{-1} & 0 \\
0 & I_{p}
\end{array}\right]\left[\begin{array}{c}
s\left(E+\bar{L}_{D} \bar{C}\right)-\bar{A} \\
\bar{C}
\end{array}\right]=\operatorname{rank}\left[\begin{array}{c}
s\left(E+\bar{L}_{D} \bar{C}\right)-\bar{A} \\
\bar{C}
\end{array}\right] .
$$

In addition, it can be verified that

$$
\operatorname{rank}\left[s\left(E+\bar{L}_{D} \bar{C}\right)-\bar{A}\right]=\operatorname{rank}\left[\begin{array}{cc}
s I_{n}-A & 0 \\
s W C & s W+I_{p}
\end{array}\right]=\operatorname{rank}\left[\begin{array}{cc}
s I_{n}-A & 0 \\
0 & I_{p}
\end{array}\right] .
$$

Together with (16) and (17), one has

$$
\operatorname{rank}\left[\begin{array}{c}
s I_{n+p}-\bar{S}^{-1} \bar{A} \\
\bar{C}
\end{array}\right]=\operatorname{rank}\left[\begin{array}{cc}
s I_{n}-A & 0 \\
0 & I_{p} \\
C & I_{p}
\end{array}\right]=\operatorname{rank}\left[\begin{array}{c}
s I_{n}-A \\
C
\end{array}\right]+p .
$$


Reminding Assumption 2.1 and Assumption 2.2, one gets

$$
\operatorname{rank}\left[\begin{array}{c}
s I_{n+p}-\bar{S}^{-1} \bar{A} \\
\bar{C}
\end{array}\right]=n+p .
$$

Hence, it can be concluded that the pair $\left(\bar{S}^{-1} \bar{A}, \bar{C}\right)$ is observable, which implies that there exists a matrix $L^{*}$ such that $-\bar{S}^{-1} \bar{A}-L^{*} \bar{C}$ is a Hurwitz matrix. Then, there exists a positive constant $\mu$ such that $-\mu I-$ $\bar{S}^{-1} \bar{A}-L^{*} \bar{C}$ is a Hurwitz matrix as well, which yields that the pair $\left(-\mu I-\bar{S}^{-1} \bar{A}, \bar{C}\right)$ is observable.

Therefore, by Lemma 2.1, there exists a matrix $X>0$ such that

$$
-\left(\mu I+\bar{S}^{-1} \bar{A}\right)^{T} X-X\left(\mu I+\bar{S}^{-1} \bar{A}\right)=-\bar{C}^{T} \bar{C} .
$$

From the above equation, the observer gain matrix can be designed as $\bar{L}_{p}=\bar{S} X^{-1} \bar{C}^{T}$, which leads to

$$
\left(\mu I+\bar{S}^{-1}\left(\bar{A}-\bar{L}_{p} \bar{C}\right)\right)^{T} X+X\left(\mu I+\bar{S}^{-1}\left(\bar{A}-\bar{L}_{p} \bar{C}\right)\right)=-\bar{C}^{T} \bar{C} .
$$

According to Lemma 2.1, it is clear that $\operatorname{Re}\left[\lambda_{i}\left(\bar{S}^{-1}\left(\bar{A}-\bar{L}_{p} \bar{C}\right)\right)\right]<-\mu, \forall i \in\{1,2, \cdots, n+p\}$, which means that $\bar{S}^{-1}\left(\bar{A}-\bar{L}_{p} \bar{C}\right)$ is a Hurwitz matrix.

\section{Adaptive controller design and stability analysis}

First, one can deduce from (3) that

$$
\begin{aligned}
\bar{S} \dot{\bar{x}} & =\left(E+\bar{L}_{D} \bar{C}\right) \dot{\bar{x}} \\
& =\bar{A} \bar{x}+\bar{B} \rho u+F g(x)+\bar{N} f_{s}+\bar{L}_{D} \bar{C} \dot{\bar{x}} \\
& =\left(\bar{A}-\bar{L}_{p} \bar{C}\right) \bar{x}+\bar{B} \rho u+\bar{L}_{p} y+F g(x)+\bar{N} f_{s}+\bar{L}_{D} \dot{y} .
\end{aligned}
$$

In addition, it follows from (11) that

$$
\begin{aligned}
\bar{S} \dot{\hat{\bar{x}}} & =\bar{S}\left(\dot{\bar{z}}+\bar{S}^{-1} \bar{L}_{D} \dot{y}\right) \\
& =\left(\bar{A}-\bar{L}_{p} \bar{C}\right) \hat{\bar{x}}+\bar{B} \hat{\rho} u+\bar{L}_{p} y+F g(\hat{x})+\bar{N} u_{s}+\bar{L}_{D} \dot{y} .
\end{aligned}
$$

Thus, by denoting $\bar{e}=\hat{\bar{x}}-\bar{x}$ and $\tilde{\rho}=\rho-\hat{\rho}$, the dynamic of error system becomes

$$
\dot{\bar{e}}=\bar{S}^{-1}\left(\bar{A}-\bar{L}_{p} \bar{C}\right) \bar{e}-\bar{S}^{-1} \bar{B} \tilde{\rho} u+\bar{S}^{-1} \bar{N} u_{s}-\bar{S}^{-1} \bar{N} f_{s}+\bar{S}^{-1} F(g(\hat{x})-g(x)) .
$$

In addition, it is derived from (23) that

$$
\dot{\hat{x}}=A \hat{x}-\bar{L}_{p_{1}} \bar{C} \bar{e}+B \hat{\rho} u+g(\hat{x}),
$$

where $\hat{x} \in R^{n}$ is the estimation of system state $x$ and $\bar{L}_{P_{1}} \in R^{n \times p}$ satisfies $\bar{L}_{p}=\left[\bar{L}_{p_{1}}^{T}, \bar{L}_{p_{2}}^{T}\right]^{T}$. Now, it is ready to provide the main theorem. 
Theorem 5.1 If there exist matrices $P \in R^{(n+p) \times(n+p)}>0, Q \in R^{n \times n}>0, H_{1} \in R^{p \times p}, H_{2} \in R^{m \times p}, K \in R^{m \times n}$, $F_{1} \in R^{n \times(n+p)}$ and scalars $\varepsilon_{1}, \varepsilon_{2}>0$ satisfying

$$
\begin{aligned}
& \Omega_{1}=\left[\begin{array}{cc}
P \bar{S}^{-1}\left(\bar{A}-\bar{L}_{p} \bar{C}\right)+\left(\bar{A}-\bar{L}_{p} \bar{C}\right)^{T} \bar{S}^{-T} P+\varepsilon_{1} I_{n+p} & L_{f} F_{1}^{T} F^{T} \bar{S}^{-T} P \\
* & -\varepsilon_{1} I_{n+p}
\end{array}\right]<0, \\
& \Omega_{2}=\left[\begin{array}{cc}
A U+U A^{T}+B Y+Y^{T} B^{T}+L_{f}^{2} \varepsilon_{2} I_{n} & U \\
* & -\varepsilon_{2} I_{n}
\end{array}\right]<0 \\
& \bar{N}^{T} \bar{S}^{-T} P=H_{1} \bar{C} \\
& \bar{B}^{T} \bar{S}^{-T} P=H_{2} \bar{C}
\end{aligned}
$$

where $U=Q^{-1}$ and $Y=K Q^{-1}$, then by designing the adaptive control law and the discontinuous input as

$$
\begin{aligned}
u= & \hat{\rho}^{-1} K \hat{x}, \\
\dot{\hat{\rho}}_{k}= & \operatorname{Proj}\left[\underline{\rho}_{k}, \bar{\rho}_{k}\right]\left\{-\gamma_{k} u_{k} M_{k} H_{2} \bar{C} \bar{e}\right\} \\
& =\left\{\begin{array}{l}
0, \text { if } \hat{\rho}_{k}=\underline{\rho}_{k},-\gamma_{k} u_{k} M_{k} H_{2} \bar{C} \bar{e} \leq 0 \\
0, \text { if } \hat{\rho}_{k}=\bar{\rho}_{k},-\gamma_{k} u_{k} M_{k} H_{2} \bar{C} \bar{e} \geq 0 \\
-\gamma_{k} u_{k} M_{k} H_{2} \bar{C} \bar{e}, \text { Otherwise }
\end{array}\right. \\
u_{s}= & -\alpha \cdot \operatorname{sign}\left(H_{1} \bar{C} \bar{e}\right),
\end{aligned}
$$

where $M_{k}=(\underbrace{0, \cdots, 0,1}_{k}, 0, \cdots, 0) \in R^{1 \times m}, \gamma_{k}>0, k=1, \cdots, m$, system state and sensor noise of system (3) can be estimated simultaneously and system state can be asymptotically regulated to the origin.

Proof: Since $\bar{S}^{-1}\left(\bar{A}-\bar{L}_{p} \bar{C}\right)$ is a Hurwitz matrix, construct a Lyapunov function $V_{\bar{e}}=\bar{e}^{T} P \bar{e}$ for system (24). The time derivative of $V_{\bar{e}}$ along the trajectory of system (24) becomes

$$
\begin{aligned}
\dot{V}_{\bar{e}}= & \bar{e}^{T}\left(P \bar{S}^{-1}\left(\bar{A}-\bar{L}_{p} \bar{C}\right)+\left(\bar{A}-\bar{L}_{p} \bar{C}\right)^{T} \bar{S}^{-T} P\right) \bar{e}-2 \bar{e}^{T} P \bar{S}^{-1} \bar{B} \tilde{\rho} u+2 \bar{e}^{T} P \bar{S}^{-1} \bar{N}\left(u_{s}-f_{s}\right) \\
& +2 \bar{e}^{T} P \bar{S}^{-1} F(g(\hat{x})-g(x)) .
\end{aligned}
$$

Next, the last two terms in the right-hand side of (33) will be estimated. According to (28), (32) and Assumption 2.3, it is not difficult to deduce that

$$
\begin{aligned}
2 \bar{e}^{T} P \bar{S}^{-1} \bar{N}\left(u_{s}-f_{s}\right) & =-2 \alpha \bar{e}^{T} P \bar{S}^{-1} \bar{N} \operatorname{sign}\left(H_{1} \bar{C} \bar{e}\right)-2 \bar{e}^{T} P \bar{S}^{-1} \bar{N} f_{s} \\
& \leq-2 \alpha \bar{e}^{T} P \bar{S}^{-1} \bar{N} \operatorname{sign}\left(\bar{N}^{T} \bar{S}^{-T} P \bar{e}\right)+2 \alpha\left|\bar{e}^{T} P \bar{S}^{-1} \bar{N}\right| \\
& \leq-2 \alpha\left|\bar{e}^{T} P \bar{S}^{-1} \bar{N}\right|+2 \alpha\left|\bar{e}^{T} P \bar{S}^{-1} \bar{N}\right| \\
& \leq 0
\end{aligned}
$$


In addition, Assumption 2.4 implies that the nonlinear terms satisfy the Lipschitz condition. So for a scalar $\varepsilon_{1}>0$, one has

$$
\begin{aligned}
2 \bar{e}^{T} P \bar{S}^{-1} F(g(\hat{x})-g(x)) & \leq \varepsilon_{1} \bar{e}^{T} \bar{e}+\frac{1}{\varepsilon_{1}}(g(\hat{x})-g(x))^{T} F^{T} \bar{S}^{-T} P P \bar{S}^{-1} F(g(\hat{x})-g(x)) \\
& \leq \varepsilon_{1} \bar{e}^{T} \bar{e}+\frac{L_{f}^{2}}{\varepsilon_{1}} \bar{e}^{T} F_{1}^{T} F^{T} \bar{S}^{-T} P P \bar{S}^{-1} F F_{1} \bar{e}
\end{aligned}
$$

where $\hat{x}-x=F_{1} \bar{e}$. Substituting (34) and (35) into (33), it yields

$$
\dot{V}_{\bar{e}} \leq \bar{e}^{T} \Pi_{1} \bar{e}-2 \bar{e}^{T} P \bar{S}^{-1} \bar{B} \tilde{\rho} u
$$

where $\Pi_{1}=P \bar{S}^{-1}\left(\bar{A}-\bar{L}_{p} \bar{C}\right)+\left(\bar{A}-\bar{L}_{p} \bar{C}\right)^{T} \bar{S}^{-T} P+\varepsilon_{1} I_{n+p}+\frac{L_{f}^{2}}{\varepsilon_{1}} F_{1}^{T} F^{T} \bar{S}^{-T} P P \bar{S}^{-1} F F_{1}$.

Next, by designing a Lyapunov function $V_{t}=\eta V_{\bar{e}}+\hat{x}^{T} Q \hat{x}+\eta \sum_{k=1}^{m} \frac{\tilde{\rho}_{k}^{2}}{\gamma_{k}}$ where $\eta>0$ is a constant to be determined later, it follows from (25), (30) and (36) that

$$
\begin{aligned}
\dot{V}_{t} \leq & \bar{e}^{T} \Pi_{1} \bar{e}-2 \eta \bar{e}^{T} P \bar{S}^{-1} \bar{B} \tilde{\rho} u+\hat{x}^{T}\left(Q(A+B K)+(A+B K)^{T} Q\right) \hat{x} \\
& -2 \hat{x}^{T} Q \bar{L}_{p_{1}} \bar{C} \bar{e}+2 \hat{x}^{T} Q g(\hat{x})-2 \eta \sum_{k=1}^{m} \frac{\tilde{\rho}_{k} \dot{\hat{\rho}}_{k}}{\gamma_{k}} .
\end{aligned}
$$

In what follows, we estimate some terms in the right-hand side of (37). First, in case of $\dot{\hat{\rho}}_{k}=0, k=1, \cdots, m$, one has

$$
\begin{aligned}
-2 \eta \bar{e}^{T} P \bar{S}^{-1} \bar{B} \tilde{\rho} u-2 \eta \sum_{k=1}^{m} \frac{\tilde{\rho}_{k} \dot{\hat{\rho}}_{k}}{\gamma_{k}} & =-2 \eta \bar{e}^{T} \bar{C}^{T} H_{2}^{T} \tilde{\rho} u \\
& =-2 \eta u^{T} \tilde{\rho} H_{2} \bar{C} \bar{e} \\
& =-2 \eta \sum_{k=1}^{m} u_{k}\left(\rho_{k}-\hat{\rho}_{k}\right) M_{k} H_{2} \bar{C} \bar{e}
\end{aligned}
$$

If $\hat{\rho}_{k}=\underline{\rho}_{k}$ and $u_{k} M_{k} H_{2} \bar{C} \bar{e} \geq 0$ or $\hat{\rho}_{k}=\bar{\rho}_{k}$ and $u_{k} M_{k} H_{2} \bar{C} \bar{e} \leq 0$, (38) becomes

$$
-2 \eta \bar{e}^{T} P \bar{S}^{-1} \bar{B} \tilde{\rho} u-2 \eta \sum_{k=1}^{m} \frac{\tilde{\rho}_{k} \dot{\hat{\rho}}_{k}}{\gamma_{k}} \leq 0 .
$$

Otherwise, in case of $\dot{\hat{\rho}}_{k}=-\gamma_{k} u_{k} M_{k} H_{2} \bar{C} \bar{e}$, it can be verified that

$$
\begin{aligned}
-2 \eta \bar{e}^{T} P \bar{S}^{-1} \bar{B} \tilde{\rho} u-2 \eta \sum_{k=1}^{m} \frac{\tilde{\rho}_{k} \dot{\hat{\rho}}_{k}}{\gamma_{k}} & =-2 \eta u^{T} \tilde{\rho} \bar{B}^{T} \bar{S}^{-T} P \bar{e}+2 \eta \sum_{k=1}^{m} \tilde{\rho}_{k} u_{k} M_{k} H_{2} \bar{C} \bar{e} \\
& =-2 \eta u^{T} \tilde{\rho} H_{2} \bar{C} \bar{e}+2 \eta \sum_{k=1}^{m} \tilde{\rho}_{k} u_{k} M_{k} H_{2} \bar{C} \bar{e} \\
& =-2 \eta \sum_{k=1}^{m} \tilde{\rho}_{k} u_{k} M_{k} H_{2} \bar{C} \bar{e}+2 \eta \sum_{k=1}^{m} \tilde{\rho}_{k} u_{k} M_{k} H_{2} \bar{C} \bar{e} \\
& =0 .
\end{aligned}
$$


Therefore, under the adaptive law (31), the following expression is guaranteed:

$$
-2 \eta \bar{e}^{T} P \bar{S}^{-1} \bar{B} \tilde{\rho} u-2 \eta \sum_{k=1}^{m} \frac{\tilde{\rho}_{k} \dot{\hat{\rho}}_{k}}{\gamma_{k}} \leq 0 .
$$

In addition, according to Assumption 2.4, there exists a positive constant $\varepsilon_{2}>0$ such that

$$
2 \hat{x}^{T} Q g(\hat{x}) \leq \frac{1}{\varepsilon_{2}} \hat{x}^{T} \hat{x}+\varepsilon_{2} g^{T}(\hat{x}) Q Q g(\hat{x}) \leq \frac{1}{\varepsilon_{2}} \hat{x}^{T} \hat{x}+L_{f}^{2} \varepsilon_{2} \hat{x}^{T} Q Q \hat{x}
$$

Substituting (41) and (42) into (37), it yields

$$
\dot{V}_{t} \leq \eta \bar{e}^{T} \Pi_{1} \bar{e}+\hat{x}^{T} \Pi_{2} \hat{x}+d_{1}\|\hat{x}\|\|\bar{e}\|
$$

where $d_{1}=2\left\|Q \bar{L}_{p_{1}} \bar{C}\right\|$ and $\Pi_{2}=Q(A+B K)+(A+B K)^{T} Q+\frac{1}{\varepsilon_{2}} I_{n}+L_{f}^{2} \varepsilon_{2} Q Q$.

By Schur's complement Lemma, if $\Omega_{1}, \Omega_{2}<0$, then $\Pi_{1}<0, \Pi_{2}<0$. Let $\sigma_{1}=-\lambda_{\max }\left\{\Pi_{1}\right\}>0$ and $\sigma_{2}=-\lambda_{\max }\left\{\Pi_{2}\right\}>0$, one has

$$
\begin{aligned}
\dot{V}_{t} & \leq-\sigma_{1} \eta\|\bar{e}\|^{2}-\sigma_{2}\|\hat{x}\|^{2}+\frac{\sigma_{2}}{2}\|\hat{x}\|^{2}+\frac{d_{1}^{2}}{2 \sigma_{2}}\|\bar{e}\|^{2} \\
& \leq-\left(\sigma_{1} \eta-\frac{d_{1}^{2}}{2 \sigma_{2}}\right)\|\bar{e}\|^{2}-\frac{\sigma_{2}}{2}\|\hat{x}\|^{2} .
\end{aligned}
$$

By choosing $\eta>\frac{d_{1}^{2}}{2 \sigma_{1} \sigma_{2}}$ and $\beta=\min \left\{\sigma_{1} \eta-\frac{d_{1}^{2}}{2 \sigma_{2}}, \frac{\sigma_{2}}{2}\right\}$, one has

$$
\dot{V}_{t} \leq-\beta\left(\|\bar{e}\|^{2}+\|\hat{x}\|^{2}\right)<0, \text { for } \forall \bar{e} \neq 0, \forall \hat{x} \neq 0 .
$$

The above inequality implies that

$$
\lim _{t \rightarrow+\infty} \bar{x}(t)=\lim _{t \rightarrow+\infty} \hat{\bar{x}}(t), \lim _{t \rightarrow+\infty} \hat{x}(t)=0 .
$$

By the definitions of $\bar{x}$ and $\hat{\bar{x}}$, it can be verified that

$$
\lim _{t \rightarrow+\infty} x(t)=\lim _{t \rightarrow+\infty} \hat{x}(t)=0, \lim _{t \rightarrow+\infty} f_{s}(t)=\lim _{t \rightarrow+\infty} \hat{f}_{s}(t),
$$

which illustrates that under the proposed adaptive control law, system state and sensor noise can be globally asymptotically estimated simultaneously. Besides, system state can be globally asymptotically regulated to the origin. This completes the proof.

Remark 5.1 It should be pointed out that the condition proposed in Theorem 5.1 includes two linear matrix equalities, which are difficult to directly solve with the Matlab tool-box. Inspired by [17], the linear equalities (28) and (29) can be rewritten as

$$
\begin{gathered}
\operatorname{Trace}\left\{\left(\bar{N}^{T} \bar{S}^{-T} P-H_{1} \bar{C}\right)^{T}\left(\bar{N}^{T} \bar{S}^{-T} P-H_{1} \bar{C}\right)\right\}=0, \\
\operatorname{Trace}\left\{\left(\bar{B}^{T} \bar{S}^{-T} P-H_{2} \bar{C}\right)^{T}\left(\bar{B}^{T} \bar{S}^{-T} P-H_{2} \bar{C}\right)\right\}=0 .
\end{gathered}
$$


Now, we introduce the following inequalities

$$
\begin{aligned}
& \left(\bar{N}^{T} \bar{S}^{-T} P-H_{1} \bar{C}\right)^{T}\left(\bar{N}^{T} \bar{S}^{-T} P-H_{1} \bar{C}\right)<\eta_{1} I_{n+p}, \\
& \left(\bar{B}^{T} \bar{S}^{-T} P-H_{2} \bar{C}\right)^{T}\left(\bar{B}^{T} \bar{S}^{-T} P-H_{2} \bar{C}\right)<\eta_{2} I_{n+p}
\end{aligned}
$$

where $\eta_{1}>0, \eta_{2}>0$ are the parameters involved with the optimization problem. By using Schur's complement lemma, (50) and (51) are equivalent to

$$
\left[\begin{array}{cc}
-\eta_{1} I_{n+p} & \left(\bar{N}^{T} \bar{S}^{-T} P-H_{1} \bar{C}\right)^{T} \\
\star & -I_{m}
\end{array}\right]<0,\left[\begin{array}{cc}
-\eta_{2} I_{n+p} & \left(\bar{B}^{T} \bar{S}^{-T} P-H_{2} \bar{C}\right)^{T} \\
\star & -I_{m}
\end{array}\right]<0 .
$$

Therefore, the design problem of (26), (27), (28) and (29) are converted into a problem of finding a global solution of the following minimization problem:

$\min \eta_{1}$ and $\min \eta_{2}$, subject to (26), (27) and (52),

which is a minimization problem that can be addressed by using the Solvers mincx in the LMI toolbox of Matlab.

\section{An illustrating example}

Consider a flexible joint robot link problem studied in $[12,23,25]$. The system nonlinearities are due to joint flexibility, modeled as a stiffening torsional spring and the gravitational force. By denoting the motor, link position and velocities as $\theta_{m}, w_{m}, \theta_{1}$ and $w_{1}$, respectively, the dynamic equations are given by

$$
\begin{aligned}
\dot{\theta}_{m} & =w_{m}, \\
\dot{w}_{m} & =\frac{k}{J_{m}}\left(\theta_{1}-\theta_{m}\right)-\frac{b}{J_{m}} w_{m}+\frac{K_{\tau}}{J_{m}} \rho u, \\
\dot{\theta}_{1} & =w_{1}, \\
\dot{w}_{1} & =-\frac{k}{J_{1}}\left(\theta_{1}-\theta_{m}\right)-\frac{m g b}{J_{1}} \sin \left(\theta_{1}\right), \\
y & =\left(\theta_{m}, w_{m}, \theta_{1}\right)^{T}+f_{s}
\end{aligned}
$$

where $J_{m}$ and $J_{1}$ are the inertia of the motor and the link, respectively. $k, b, K_{\tau}, m$ and $g$ are system parameters. The unknown parameter $\rho$ implies that actuator degradation occurs and $f_{s} \in R^{3}$ is the unknown sensor noise. The two unknown parameters are assumed to satisfy

$$
0.2 \leq \rho \leq 0.8, f_{s}=\left[\begin{array}{c}
1+0.2 \sin (3 t) \\
0.5 \cos (t) \\
2+0.1 \sin (t)-0.3 \cos (2 t)
\end{array}\right]
$$


Then, by denoting

$$
\begin{gathered}
x=\left[\begin{array}{l}
\theta_{m} \\
w_{m} \\
\theta_{1} \\
w_{1}
\end{array}\right], A=\left[\begin{array}{cccc}
0 & 1 & 0 & 0 \\
-48.6 & -1.25 & 48.6 & 0 \\
0 & 0 & 0 & 10 \\
1.95 & 0 & -1.95 & 0
\end{array}\right], B=\left[\begin{array}{c}
0 \\
21.6 \\
0 \\
0
\end{array}\right], \\
C=\left[\begin{array}{llll}
1 & 0 & 0 & 0 \\
0 & 1 & 0 & 0 \\
0 & 0 & 1 & 0
\end{array}\right], g(x)=\left[\begin{array}{c}
0 \\
0 \\
0 \\
-0.1 \sin \left(\theta_{1}\right)
\end{array}\right]
\end{gathered}
$$

system (53) is equivalent to

$$
\begin{aligned}
& \dot{x}=A x+B \rho u+g(x), \\
& y=C x+f_{s} .
\end{aligned}
$$

By a simple calculation, it is obvious to find that Assumptions 2.1, 2.2, 2.3 and 2.4 hold with $\alpha=4.1$ and $L_{f}=0.1$. Therefore, by choosing

$$
\bar{L}_{D}=\left[\begin{array}{ccccccc}
0 & 0 & 0 & 0 & 0.5 & 0 & 0 \\
0 & 0 & 0 & 0 & 0 & 0.5 & 0 \\
0 & 0 & 0 & 0 & 0 & 0 & 0.5
\end{array}\right]
$$

the matrices $\bar{S}$ and $\bar{S}^{-1}$ are designed as

$$
\bar{S}=\left[\begin{array}{ccccccc}
1 & 0 & 0 & 0 & 0 & 0 & 0 \\
0 & 1 & 0 & 0 & 0 & 0 & 0 \\
0 & 0 & 1 & 0 & 0 & 0 & 0 \\
0 & 0 & 0 & 1 & 0 & 0 & 0 \\
0.5 & 0 & 0 & 0 & 0.5 & 0 & 0 \\
0 & 0.5 & 0 & 0 & 0 & 0.5 & 0 \\
0 & 0 & 0.5 & 0 & 0 & 0 & 0.5
\end{array}\right], \bar{S}^{-1}=\left[\begin{array}{ccccccc}
1 & 0 & 0 & 0 & 0 & 0 & 0 \\
0 & 1 & 0 & 0 & 0 & 0 & 0 \\
0 & 0 & 1 & 0 & 0 & 0 & 0 \\
0 & 0 & 0 & 1 & 0 & 0 & 0 \\
-1 & 0 & 0 & 0 & 2 & 0 & 0 \\
0 & -1 & 0 & 0 & 0 & 2 & 0 \\
0 & 0 & -1 & 0 & 0 & 0 & 2
\end{array}\right] .
$$


Together with the choice of $\mu=3$, one can deduce from (20) and (58) that

$$
\bar{L}_{P}=\bar{S} X^{-1} \bar{C}^{T}=\left[\begin{array}{ccc}
6.4766 & 7.8721 & 7.1304 \\
-2.3655 & 62.8851 & 14.7437 \\
6.5065 & 5.9313 & 8.2946 \\
0.2584 & -0.9343 & -0.0111 \\
2.5885 & 0.1226 & 1.5329 \\
0.1226 & 8.3818 & 0.9988 \\
1.5329 & 0.9988 & 2.7797
\end{array}\right] .
$$

According to Theorem 5.1, the parameters $K, H_{1}, H_{2}$ are calculated and designed as

$$
\begin{aligned}
& K=\left[\begin{array}{llll}
-294.1346 & -67.5851 & 114.4186 & -207.1827
\end{array}\right], \\
& H_{1}=\left[\begin{array}{ccc}
0.002 & -3.1684 \times 10^{-5} & 6.8485 \times 10^{-4} \\
-1.8629 \times 10^{-4} & 6.6680 \times 10^{-4} & 8.1087 \times 10^{-5} \\
6.5904 \times 10^{-4} & 3.4365 \times 10^{-5} & 6.5771 \times 10^{-4}
\end{array}\right] \text {, } \\
& H_{2}=\left[\begin{array}{lll}
-3.483 \times 10^{-5} & -6.5025 \times 10^{-4} & -9.3276 \times 10^{-4}
\end{array}\right] \text {, }
\end{aligned}
$$

with parameters $\eta_{1}=\eta_{2}=10^{-5}$.

Hence, the novel PD observer and adaptive controller are constructed as

$$
\begin{aligned}
& \bar{S} \dot{\bar{z}}=\left(\bar{A}-\bar{L}_{p} \bar{C}\right) \hat{\bar{x}}+\bar{B} \hat{\rho} u+\bar{L}_{p} y+F g(\hat{x})-4.1 \bar{N} \operatorname{sign}\left(H_{1} \bar{C} \bar{e}\right), \\
& \hat{\bar{x}}=\bar{z}+\bar{S}^{-1} \bar{L}_{D} y \\
& u=\hat{\rho}^{-1} K \hat{x}, \\
& \dot{\hat{\rho}}=\operatorname{Proj}[0.2,0.8]\left\{-u H_{2} \bar{C} \bar{e}\right\} \\
&=\left\{\begin{array}{l}
0, \text { if } \hat{\rho}=0.2,-u H_{2} \bar{C} \bar{e} \leq 0 \\
0, \text { if } \hat{\rho}=0.8,-u H_{2} \bar{C} \bar{e} \geq 0 \\
-u H_{2} \bar{C} \bar{e}, \text { Otherwise }
\end{array}\right.
\end{aligned}
$$

where matrices $\bar{A}, \bar{B}, \bar{C}, F, \bar{N}$ are defined in (8) with $p=3, n=4$ and $m=1$.

In the simulation, the system parameter $\rho$ is chosen as $\rho=0.5$ and the initial conditions are $x(0)=$ $[0.5,1,1.3,0.6]^{T}, \bar{z}(0)=[0,0,0,0,0,0,0]^{T}$ and $\hat{\rho}(0)=0.3$. It is shown in Figure 1 and Figure 2 that under the method in [17], the system state is unstable and sensor noise can not be reconstructed. By the proposed adaptive control law, the above drawbacks are overcome and system state can be regulated to the origin 
asymptotically. Figure 3 shows the trajectory of control input by the proposed method and the method in [17]. Figures 1-3 illustrate that the effectiveness of our proposed method despite the appearance of sensor noise and actuator degradation.

\section{Conclusion}

This paper has addressed the control problem of nonlinear systems with sensor noise and actuator degradation via output feedback. By developing a novel PD observer and an adaptive observer-based controller, system state and sensor noise can be reconstructed simultaneously and system state can be regulated to the origin asymptotically. The design procedure is simple and avoids discussing the reachability of sliding mode surface. Besides, the proposed design scheme is not limited to triangular structure systems.

\section{Acknowledgements}

This work is supported by the National Science Foundation of China under Grant $(61273119,61473283)$. Besides, it is funded by State Key Laboratory of Robotics (Grant No. 2014-O08). It is also funded by Changshu Institute of Technology under Grant (KYZ2015046Z).

\section{References}

[1] Jo HW, Choi HL, Lim JT. Measurement feedback control for a class of feedforward nonlinear systems. International Journal of Robust and Nonlinear Control 2013; 23(12): 1405-18.

[2] Jo HW, Choi HL, Lim JT. Output feedback control of feedforward nonlinear systems in the presence of sensor noise. International Journal of Robust and Nonlinear Control 2014; 24(13): 1845-57.

[3] Khalil HK. Analysis of sampled-data high-gain observers in the presence of measurement noise. European Journal of Control 2009; 15(2): 166-76.

[4] Ahrens JH, Khalil HK. High-gain observers in the presence of measurement noise: A switched-gain approach. Automatica 2009; 45(4), 936-43.

[5] Prasov AA, Khalil HK. A nonlinear high-gain observer for systems with measurement noise in a feedback control framework. IEEE Transactions on Automatic Control 2013; 58(3): 569-80. 

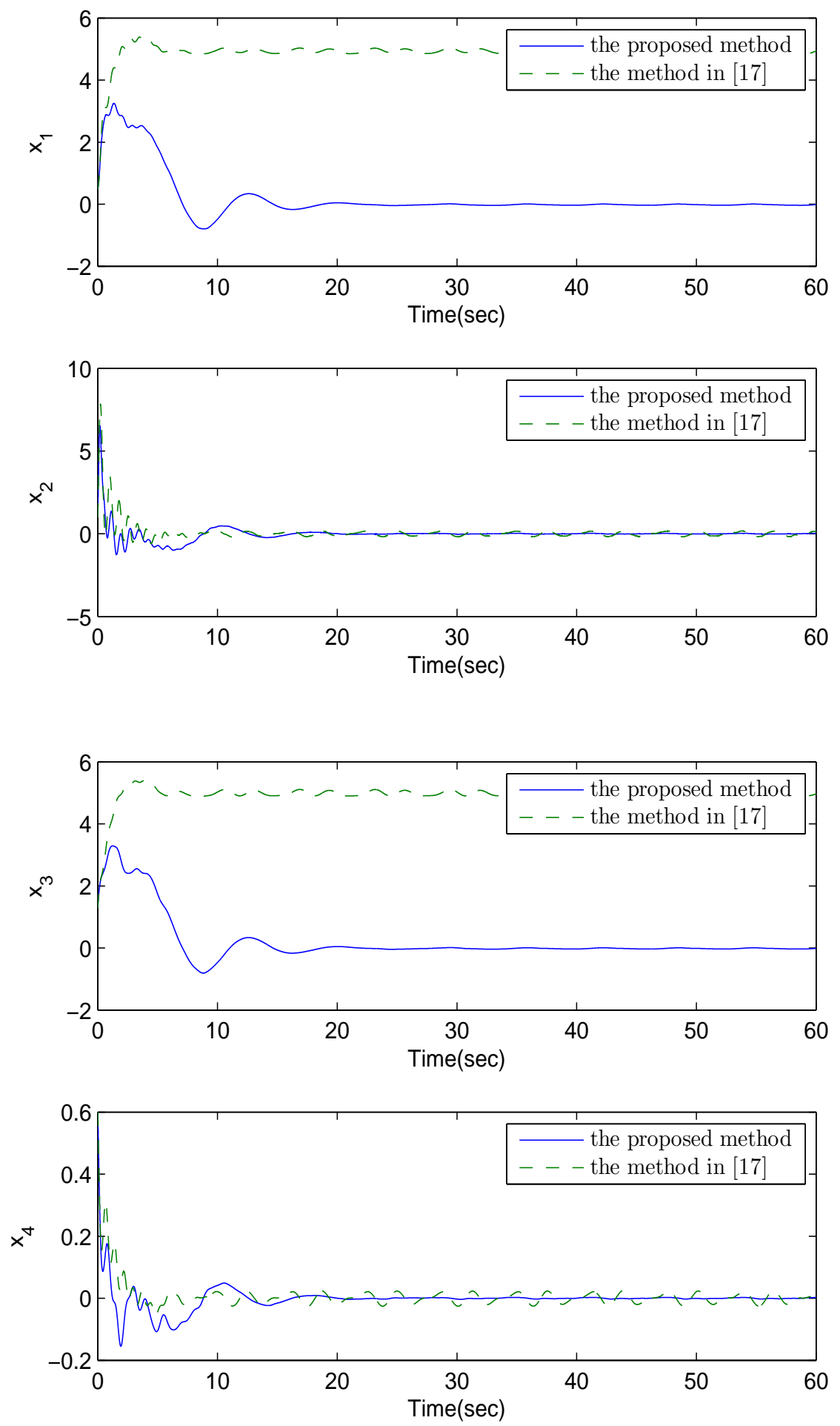

Figure 1: Trajectories of system state $x$ under the proposed method and the method in [17] 

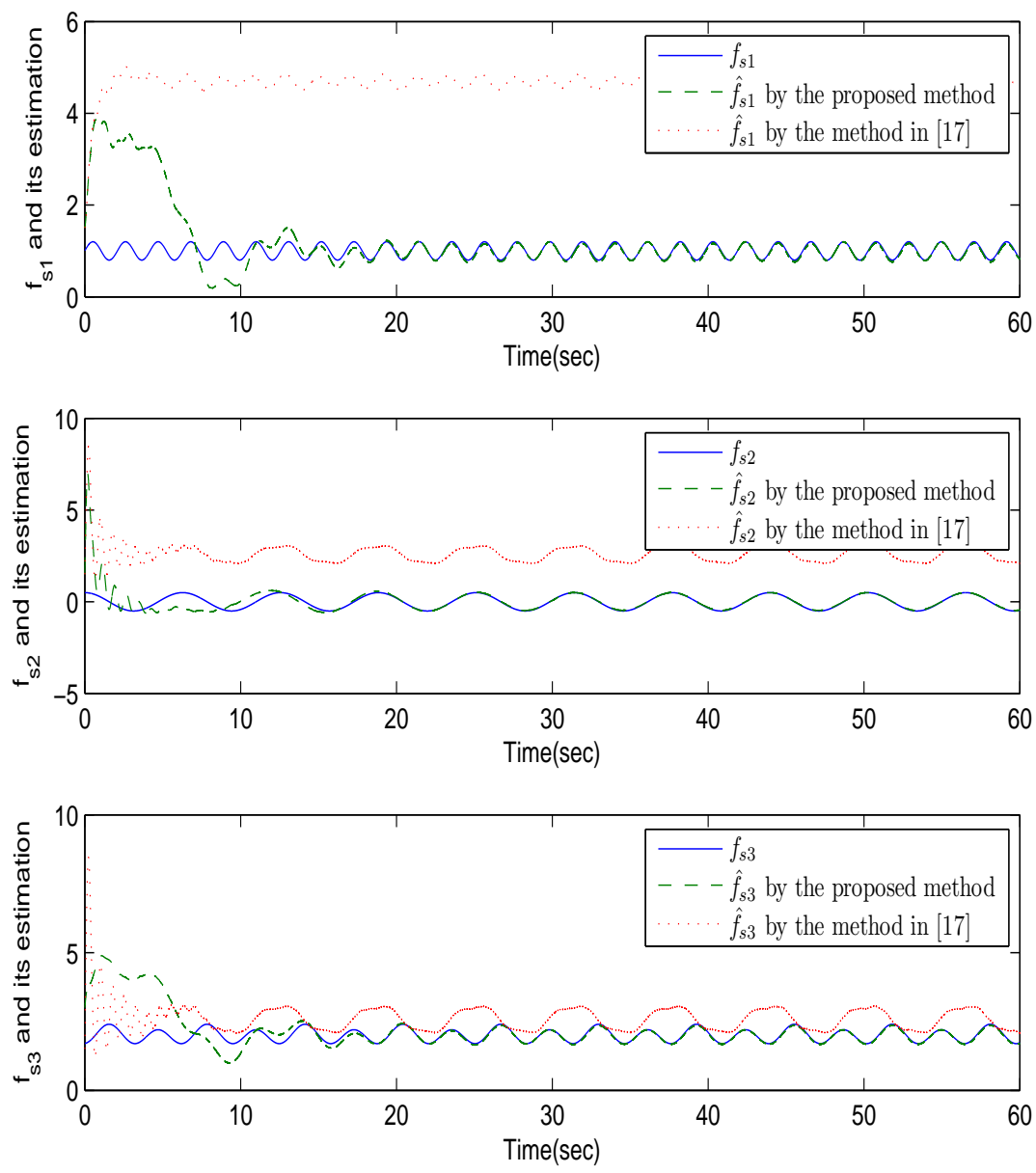

Figure 2: Trajectories of sensor noise and its estimation by the proposed method and the method in [17]

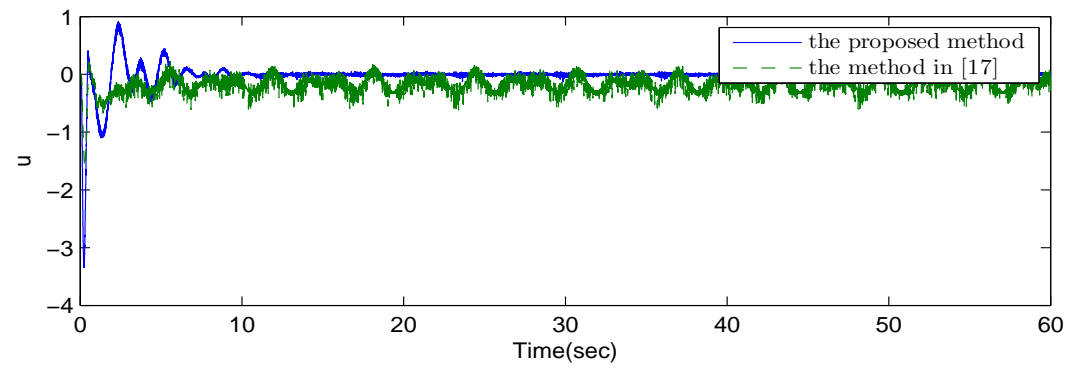

Figure 3: Trajectories of control input by the proposed method and the method in [17] 
[6] Sanfelice RG, Praly L. On the performance of high-gain observers with gain adaptation under measurement noise. Automatica 2011; 47(10), 2165-76.

[7] Vijayaraghavan K. Generalised observer design for dissipative Lipschitz nonlinear systems in the presence of measurement noise. International Journal of Control 2014; 87(11): 2273-85.

[8] Vijayaraghavan K. Observer design for generalized sector-bounded noisy nonlinear systems. Proceedings of the Institution of Mechanical Engineers, Part I: Journal of Systems and Control Engineering, 2014; 228(9): 645-57.

[9] Yang JQ, Zhu FL, Zhang W. Sliding-mode observers for nonlinear systems with unknown inputs and measurement noise. International Journal of Control, Automation and Systems 2013; 11(5): 903-10.

[10] Yang JQ, Chen YT, Zhu FL. Singular reduced-order observer-based synchronization for uncertain chaotic systems subject to channel disturbance and chaos-based secure communication. Applied Mathematics and Computation 2014; 229(6): 227-38.

[11] Zhang J, Swain AK, Nguang SK. Robust sensor fault estimation scheme for satellite attitude control systems. Journal of the Fraklin Institute 2013; 350(9): 2581-04.

[12] Zhang J, Swain AK, Nguang SK. Simultaneous robust actuator and sensor fault estimation for uncertain non-linear Lipschitz systems. IET Control Theory and Applications 2014; 8(14): 1364-74.

[13] Gao ZW, Wang H. Descriptor observer approaches for multivariable systems with measurement noises and application in fault detection and diagnosis. Systems \& Control Letters 2006; 55(4): 304-13.

[14] Gao ZW, Breikin T, Wang H. High-gain estimator and fault-tolerant design with application to a gas turbine dynamic systems. IEEE Transactions on Control Systems Technology 2007; 15(4): 740-53.

[15] Liu M, Shi P, Zhang LX, Zhao XD. Fault-tolerant control for nonlinear Markovian jump systems via proportional and derivative sliding mode observer technique. IEEE Transactions on Circuits and Systems-I 2011; 58(11): 2755-64.

[16] Liu M, Cao XB, Shi P. Fuzzy-mode-based fault-tolerant design for nonlinear stochastic systems against simultaneous sensor and actuator faults. IEEE Transactions on Fuzzy Systems 2013; 21(5): 789-99.

[17] Liu M, Shi P. Sensor fault estimation and tolerant control for Itô stochastic systems with a descriptor sliding mode approach. Automatica 2013; 49(5): 1242-50. 
[18] Liu M, Zhang LX, Shi P, Karimi HR. Robust control of stochastic systems against bounded disturbances with application to flight control. IEEE Transactions on Industrial Electronics 2014; 61(3): $1504-15$.

[19] Li HY, Gao HJ, Shi P, Zhao, XD. Fault-tolerant control of Markovian jump stochastic systems via the augmented sliding mode observer approach. Automatica 2014; 50(7): 1825-34.

[20] Zhang J, Swain AK, Nguang SK. Robust H-infinity adaptive descriptor observer design for fault estimation of uncertain nonlinear systems. Journal of the Franklin Institute 2014; 351(11): 5162-81.

[21] Khebbache H, Tadjine M, Labiod S, Boulkroune A. Adaptive sensor-fault tolerant control for a class of multivariable uncertain nonlinear systems. ISA Transactions 2015; 55:100-15.

[22] Zhao KK, Jiang B, Yan XG, Mao ZH. Sliding mode observer based in cipient sensor fault detection with application to high-speed railway traction device. ISA Transactions 2016; doi:10.1016/j.isatra.2016.04.004

[23] Jiang B, Staroswiecki M, Cocquempot V. Fault accommodation for nonlinear dynamic systems. IEEE Transactions on Automatic Control 2006; 51(9): 1578-83.

[24] Ding S. Model-based fault diagnosis techniques: Design schemes. Berlin: Springer-Verlag; 2008.

[25] Zhang K, Jiang B, Shi, P. Fast fault estimation and accommodation for dynamical systems. IET Control Theory and Applications 2009; 3(2): 189-99.

[26] Zuo Z, Ho DWC, Wang Y. Fault tolerant control for singular systems with actuator saturation and nonlinear perturbation. Automatica 2010; 46(3): 569-76.

[27] Chen B, Niu YG, Zou YY. Adaptive sliding mode control for stochastic Markovian jumping systems with actuator degradation. Automatica 2013; 49(6): 1748-54.

[28] Li JZ. Distributed cooperative tracking of multi-agent systems with actuator faults. Transactions of the Institute of Measurement and Control 2015; 37(9): 1041-48.

[29] Zhong GX, Yang GH. Fault detection for discrete-time switched systems with sensor stuck faults and servo inputs. ISA Transactions 2015; 58:192-05. 
[30] Zhuang GM, Li YM, Li Z. Fault detection for a class of uncertain nonlinear Markovian jump stochastic systems with mode-dependent time delays and sensor saturation. International Journal of Systems Science 2016; 47(7): 1514-32.

[31] Cai J, Ferdowsi H, Sarangapani J. Model-based fault detection, estimation, and prediction for a class of linear distributed parameter systems. Automatica 2016; 66: 122-31.

[32] Tao G, Chen S, Joshi SM. An adaptive actuator failure compensation controller using output feedback. IEEE Transactions on Automatic Control 2002; 47(3): 506-11.

[33] Corless M, Tu J. State and input estimation for a class of uncertain systems. Automatica 1998; 34(6): 757-64.

[34] Chen C. Linear system theory and design. New York: Oxford University Press; 1999. 